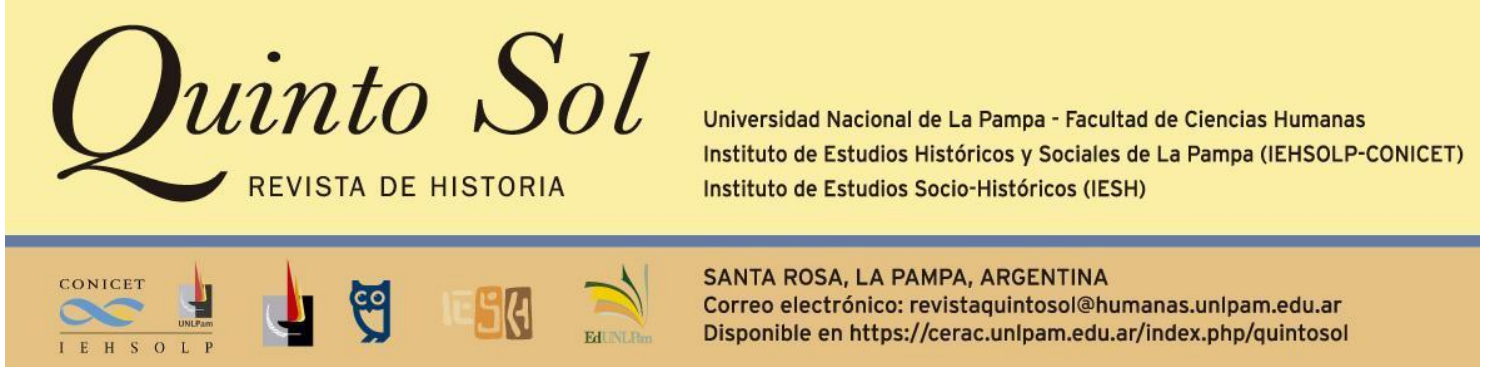

Quinto Sol, vol. 26, no 1, enero-abril 2022, ISSN 1851-2879, pp. 1-22

http://dx.doi.org/10.19137/qs.v26i1.5352

Esta obra se publica bajo licencia Creative Commons 4.0 Internacional. (Atribución-No Comercial- (c) (i) () () Compartir Igual)

\title{
La historia política de Santa Fe desde la transición democrática (1983-2020): aportes, líneas de investigación y vacancias
}

\section{Political history of Santa Fe since the democratic transition (1983-2020): contributions, lines of research and vacancies}

\section{A história política de Santa Fe desde a transição democrática (1983-2020): contribuições, linhas de investigação e vagas}

\section{María Cecilia Lascurain}

Universidad de Buenos Aires. Facultad de Ciencias Sociales

Argentina

Correo electrónico: mcecilia.lascurain@outlook.com

\section{Hugo Daniel Ramos}

Universidad Nacional del Litoral. Facultad de Humanidades y Ciencias

Argentina

Correo electrónico: ramoshugo78@gmail.com

\section{Mariano Vaschetto}

Consejo Nacional de Investigaciones Científicas y Técnicas

Universidad Nacional de Rosario. Facultad de Ciencia Política y Relaciones Internacionales. Instituto de Investigaciones

Argentina

Correo electrónico: marianovaschetto@gmail.com 
Lascurain, M.C.; Ramos, H. D. y Vaschetto, M. La historia política de Santa Fe desde la transición democrática (19832020): aportes, líneas de investigación y vacancias

\section{Resumen}

Desde un enfoque anclado en la nueva historia política, el presente trabajo analiza y sistematiza los principales aportes provenientes de las ciencias sociales referidos al conocimiento de la historia reciente de la provincia de Santa Fe, Argentina. Nos centramos en particular en las producciones que abordan el período que va desde la transición democrática hasta la actualidad (1983-2020), para destacar las principales líneas de investigación, sus contribuciones al campo y las vacancias observadas. El artículo se organiza en torno a tres ejes: el estudio de los sistemas electorales y del sistema político provincial, las contribuciones que abordan las características y dinámicas de interacción de los distintos actores político-partidarios, y los aportes sobre otros actores sociales en interacción con el sistema político local. El objetivo es brindar un panorama completo y exhaustivo que dé cuenta de la literatura relativa a la historia política reciente de Santa Fe.

\section{Abstract}

This work, grounded in the perspective of new political history, analyzes and systematizes the most relevant contributions from the social sciences to the knowledge of Santa Fe (Argentina) province's recent history. We particularly focus on works that deal with the period that goes from the democratic transition to the present day (1983-2020), highlighting the most important lines of work, their contributions to the field and the observed vacancies. The article is organized around three central issues: the study of the province's electoral and political systems; works dealing with the characteristics and interplay dynamics between political-partisan actors; and contributions regarding other social actors as they relate to the local political system. The purpose of our work is to present a complete and thorough outlook of the current knowledge available regarding Santa Fe's recent political history.

\section{Resumo}

Desde um foco que parte da nova história política, este trabalho analisa e sistematiza as principais contribuições provenientes das ciências sociais referidas ao conhecimento da história recente da província de Santa $\mathrm{Fe}$, Argentina. Centramo-nos, particularmente, nas produções do período da transição democrática até a atualidade (1983-2020), para destacar as principais linhas de investigação, suas contribuições ao campo e as vagas observadas. $\mathrm{O}$ artigo, organiza-se em três eixos: o estudo dos sistemas eleitorais e do sistema político provincial, as contribuições que tratam sobre as características e dinâmicas de interação dos diferentes atores político partidários, e as contribuições sobre outros atores sociais em interação com o sistema político local. O objetivo é oferecer um panorama completo e exaustivo que de conta da literatura relativa e da história política recente de Santa Fé.

Palavras-chave

história recente política subnacional

Recepción del original: 01 de octubre de 2020.

Aceptado para publicar: 27 de julio de 2021. 


\section{La historia política de Santa Fe desde la transición democrática (1983-2020): aportes, líneas de investigación y vacancias}

\section{Introducción}

Desde hace algunos años ha cobrado relevancia el estudio de los espacios regionales/provinciales. Con variadas tradiciones según la disciplina que consideremos, lo "subnacional", "regional" o "la escala local" se ha transformado en un objeto de creciente interés para politólogos, historiadores, sociólogos y cientistas sociales en general.

Desde la historiografía, nuestro trabajo se inscribe en la confluencia de tres campos: la nueva historia política, ${ }^{1}$ la historia reciente y la historia regional. Inicialmente indagamos en torno a las temáticas abordadas en los últimos años referidas a la provincia de Santa Fe. Con el avance de nuestra pesquisa, sin embargo, fue evidente que las producciones provenientes del campo de la ciencia política y de la sociología política eran insoslayables, en particular por la importancia que los desarrollos teóricos y las investigaciones empíricas de estas disciplinas han tenido para la producción historiográfica. Decidimos entonces elaborar un "estado de la cuestión" que, situando el foco en la historia regional en el período postransicional (1983-2020) y atendiendo a la dimensión política de los procesos históricos, nos permita dar cuenta de las vacancias, las áreas de interés y las problemáticas que han suscitado la atención de los cientistas sociales que investigan la historia reciente de Santa Fe.

Cabe destacar que no desconocemos los debates en torno al concepto de transición; apelamos en este sentido a una definición mínima: "intervalo que se extiende entre un régimen político y otro" (O’Donnell y Shmitter, 2010, p. 27) para abordar el conjunto de estudios desarrollados desde principios de los años ochenta, en el marco de la disolución de la dictadura militar y el ascenso al poder de Raúl Alfonsín hasta la actualidad. Esta decisión nos ubica también en el área de discusión acerca de la periodización de la historia reciente como campo de estudios (Franco y Levín, 2007). Recuperando el planteo de las autoras citadas, entendemos que es posible y deseable que la historiografía emprenda el análisis de los años recientes, más aún cuando no existen criterios profesionales claros que delimiten temporalmente al campo.

\footnotetext{
${ }^{1}$ Como plantean numerosos autores, la nueva historia política reconfiguró parte del campo historiográfico argentino desde la década de los ochenta y favoreció la emergencia de novedosas preguntas y líneas de indagación. Entre ellas, cabe mencionar el estudio de las dinámicas de interacción entre actores, los conflictos dentro y en relación con las instituciones, las relaciones de poder y las prácticas culturales. Asimismo, dio un fuerte impulso a las diversas historias provinciales o regionales.
} 
Finalmente, al inscribirse en la historia regional, nuestro aporte nos introduce al problema de las escalas de análisis. En sintonía con lo que plantea Gabriela Águila (2015), entendemos que indagar en la historia política de Santa Fe implica problematizar la validez de ese recorte espacial; en este caso, asumimos que estudiar las dinámicas políticas subnacionales implica distinguir las divisiones políticoadministrativas, en este caso a escala provincial, lo que no significa negar la posibilidad de reconocer otras construcciones posibles.

En esta línea, el desarrollo de nuestra investigación nos permitió advertir lo fragmentario y selectivo del conocimiento producido por las ciencias sociales en general y por la historia en particular sobre los espacios provinciales de nuestro país. Asimismo, posibilitó identificar las principales líneas de indagación, sus progresivos desarrollos y la vinculación entre la producción académica y determinadas coyunturas que habilitaron y/u obturaron el avance en las investigaciones.

El trabajo involucró un relevamiento exhaustivo de la producción académica presentada en congresos de la especialidad, revistas indexadas, capítulos de libros y libros vinculados con nuestra área de indagación. Asimismo, relevamos tesis de grado y posgrado, con especial atención hacia aquellas producidas en el ámbito de la Universidad Nacional del Litoral y la Universidad Nacional de Rosario, las dos principales universidades públicas de la provincia. Cabe destacar que, por razones de espacio, decidimos excluir de esta presentación las investigaciones referidas a las políticas públicas del Estado santafesino, un campo relativamente numeroso y claramente monopolizado por la ciencia política. Asimismo, y por idénticos motivos, solo mencionamos una selección de trabajos que pueden considerarse como significativos, ya sea por sus aportes conceptuales o por su innovación en términos teóricos y empíricos.

El texto se divide en tres apartados y las conclusiones: en primer lugar, damos cuenta de la producción referida a los sistemas electorales y al sistema político santafesino; en segundo término, abordamos los estudios que analizan las características y dinámicas de interacción de los distintos actores político-partidarios de la provincia; finalmente nos centramos en los trabajos que examinan la relación entre actores políticos y otros actores sociales. ${ }^{2}$ Cerramos con una serie de conclusiones generales.

\section{Los sistemas electorales y las investigaciones sobre el sistema político santafesino}

Las primeras investigaciones que se preguntaron sobre la especificidad de la historia política reciente santafesina se concentraron en el análisis de su sistema

\footnotetext{
${ }^{2}$ La agrupación en estas tres áreas busca clasificar la bibliografía relevada en base a su cercanía temática. Son agrupaciones provisorias y tentativas diseñadas para facilitar el análisis de la importante producción académica relevada.
} 
electoral. El puntapié inicial fue la sanción de la ley de lemas $^{3}$ (1990), que inspiró numerosas indagaciones.

Es posible establecer una periodización de estos estudios, aspecto que también permite sistematizar sus principales aportes. Los trabajos iniciales fueron contemporáneos a la sanción de la mencionada ley (Petracca, 1991; Robin, 1994), aunque el área de estudios adquirió mayor densidad a partir de la segunda mitad de la década del noventa, a medida que quedaban más claras las "consecuencias" de la legislación electoral del sistema político provincial. Dos fueron las preocupaciones centrales, que se extienden hasta la actualidad: los "efectos" sobre la estructura de la competencia partidaria y el "impacto" al interior de los partidos políticos.

Con respecto a la primera, es una conclusión compartida que la legislación tendió a estructurar un sistema partidario bipolar, en particular desde 1995, cuando la competencia política provincial se articuló en torno a dos grandes coaliciones: la comandada por el Partido Justicialista (PJ) -que gobernó la provincia entre 1983 y 2007- y la definida en torno al eje Unión Cívica Radical (UCR)-Partido Socialista (PS)Partido Demócrata Progresista (PDP). ${ }^{4}$ La categoría de "esquema bipolar" se consolidó a partir de los aportes de Ernesto Calvo y Marcelo Escolar (2005), si bien ya estaba presente como idea en los trabajos anteriores (Puig, 2000). Hasta el año 2001, el centro del esquema aliancista opositor al oficialismo provincial giró en torno de la UCR; a partir de entonces, y en consonancia con su declive electoral, es el PS el que empieza a ocupar ese lugar. Como derivación de este esquema, las investigaciones acuerdan en que las terceras fuerzas tuvieron dificultades para extender su presencia actuando en soledad, ya sea en el nivel provincial (Gery y Robin, 2005) o en la arena municipal/comunal (Fernández, 2017).

En referencia al impacto hacia el interior de las organizaciones partidarias, también existe consenso en torno a que la ley agudizó la fragmentación interna, contribuyó a diluir las identidades partidarias y favoreció la rearticulación organizativa alrededor de liderazgos electoralmente exitosos. Este último aspecto aparece como indisociable de la creciente importancia de la imagen pública para la construcción de las candidaturas y del nuevo rol de los medios de comunicación en la escena política (Delgado y Cherny, 2004). Este conjunto de cambios fue decisivo para la transformación de los partidos de masas históricos (en particular, del PJ y la UCR) en partidos "profesionales-electorales"5 (Robin, 2007).

\footnotetext{
${ }^{3}$ La Ley 10.524 instaura un procedimiento de acuerdo con el cual el elector elige un candidato (sublema) dentro de la amplia oferta presentada por los partidos o coaliciones (lemas). Es decir que, al elegir un sublema, también elige un lema. Cuando finaliza la elección, el sublema más votado suma a su favor todos los votos de los demás sublemas, que son considerados los votos totales del lema.

${ }^{4}$ Luego de las elecciones de 1983, en la provincia de Santa Fe se estructuró un esquema bipartidista de competencia política, con un tercer partido de relevancia electoral, el PDP. Hacia fines de la década del ochenta, además, se afianzó en el sur provincial el Partido Socialista Popular (PSP, luego PS), que logró acceder a la intendencia de Rosario en 1989.

${ }^{5}$ La mayor parte de estos trabajos se inscriben en la línea teórica de Bernard Manin (1996), referida a los cambios en los formatos de representación, al rol de los partidos y a sus transformaciones organizacionales.
} 
También cabe destacar que es un aspecto común en estos estudios su preocupación por la legitimidad del sistema electoral, así como su vinculación con la noción de "crisis de representación" (Petracca, Mutti, Molteni, Ford y Borello, 2003). De todas formas, y con la temprana excepción de Cristina Díaz (1996), la mayoría de los análisis entiende que la adopción de la ley de lemas fue una derivación de la fragmentación partidaria y de los escándalos de corrupción de los gobiernos justicialistas de los ochenta, más que una propiedad o característica estructural del sistema, al menos hasta el año 2001. Decíamos que la excepción es Díaz (1996), porque en esta autora se observa un esfuerzo por entender la legislación como un intento de adaptación de las organizaciones partidarias frente a cambios más profundos vinculados tanto a las transformaciones en las relaciones de representación como al reemplazo del modelo de acumulación y sus consecuencias sociopolíticas.

Ahora bien, derogada la ley de lemas en el 2004, los nuevos trabajos del área se diversificaron en dos grandes líneas: 1) aquellos que analizan los "efectos" de la Ley 12.367 o ley de Primarias Abiertas, Simultáneas y Obligatorias (PASO) ${ }^{6}$ y, posteriormente, el impacto de la boleta única ${ }^{7}$ (Mutti, Borello y Lodi, 2008; Fernández, 2017); 2) aquellos que, asumiendo nuevas perspectivas, intentan dar cuenta de otras facetas de los sistemas electorales.

Una revisión de los aportes recientes muestra que el primer grupo llega a la conclusión de que, aún sin ley de lemas, se han profundizado los procesos de fragmentación partidaria, personalización de la política y dilución de las identidades políticas históricas. Asimismo, que el esquema bipolar de competencia política se extendió hasta el 2011, aun cuando ya regía otra legislación electoral. ${ }^{8}$ Finalmente, algunos trabajos dan cuenta de que, durante la vigencia de la ley de lemas, la fragmentación interna de los partidos no se trasladó a la legislatura provincial; en contraposición con esto, desde la sanción de las PASO se han multiplicado los bloques legislativos (Tibaldo, 2013). Frente a estos resultados, es significativo entonces que no se ponga en cuestión, más que marginalmente, la idea consolidada de que el sistema electoral opera como una variable independiente que moldea tanto las estructuras organizativas de los partidos como la estructura de la competencia política provincial; de hecho, hay un amplio predominio de los enfoques definidos como institucionalistas. Encontramos aquí entonces una de las limitaciones de esta corriente de investigación, que parece no lograr dar cuenta de las complejidades de los procesos políticos provinciales y de las transformaciones operadas, con y sin ley de lemas.

Aun así, este campo de trabajo ha realizado aportes significativos, en especial referidos a la identificación de los sectores internos de los partidos desde los noventa y a sus vinculaciones con la arena nacional (Robin, 2007), al avance en torno a la

\footnotetext{
${ }^{6}$ Las PASO obligan a los partidos a dirimir sus candidaturas en internas abiertas a toda la ciudadanía (aun cuando el partido presente una sola lista) y en acto eleccionario simultáneo.

${ }^{7}$ La boleta única, sancionada en el año 2010 mediante la Ley 13.156, dispone que todas las candidaturas al mismo cargo se ubiquen en una única boleta. Hay tantas boletas como cargos en disputa.

${ }^{8}$ En ese año, el partido Propuesta Republicana (PRO) obtuvo el segundo lugar en las elecciones a gobernador en Santa $\mathrm{Fe}$, lo cual instaló una lógica de competencia a "tres bandas" que se repetiría en las elecciones sucesivas.
} 
comprensión de sus estrategias electorales (Puig, 2000) y a la clara delimitación de fenómenos de largo alcance desde una perspectiva local, como lo son la territorialización de la política y la creciente volatilidad electoral (Fernández, 2017).

La derogación de la ley de lemas habilitó la emergencia de otras líneas de trabajo, que consideramos como parte de un segundo grupo de pesquisas. Nuestra afirmación no debe interpretarse como una relación de causa-consecuencia, ya que esas líneas estaban emergiendo de forma paralela. Aquí se destacan las indagaciones sobre género; los aportes que analizan los procesos de reforma institucional y las investigaciones referidas a regiones y procesos de regionalización.

Las producciones sobre género emergen en relación con la discusión acerca de los efectos de la ley de cupo santafesina sancionada en 1992, por la cual se estableció que un tercio de las listas de candidatos a los distintos cargos locales debía estar integrada por mujeres, junto con ciertos requisitos vinculados al orden de las candidaturas. Esos aportes demuestran tanto el incremento del número de mujeres en la legislatura como el efecto reductor de los sistemas electorales ${ }^{9}$ (Ferro, 2003; Perri, 2012). Es importante mencionar que estos incorporan teorías asociadas a los análisis de género, lo que da cuenta de la desigualdad estructural entre hombres y mujeres, de la dificultad de estas para desarrollarse en el campo de la política y de los estereotipos y prejuicios que enmarcan su accionar. Asimismo, discuten con las nociones de democracia e igualdad/desigualdad y defienden la necesidad de sostener medidas de discriminación positiva. Así, su aporte fundamental se vincula con dejar en evidencia que el ejercicio del poder político en el espacio santafesino tiene género y que ese género es masculino. Esta situación es lo que ha impulsado el desarrollo de nuevas investigaciones en relación con las acciones extrainstitucionales de los movimientos de mujeres en pos de la paridad; esto es: la sanción de una legislación que asegure un reparto igualitario de las candidaturas entre hombres y mujeres (Perri, 2017; Catalin, 2019).

En lo que refiere a trabajos enfocados en los procesos de reforma institucional, se advierte un claro interés centrado en dos coyunturas específicas: la sanción de la ley de lemas en 1991 y su derogación en 2004 (Rulli y Centeno Lappas, 2006; Lodi, 2010; Toppi, 2015). Estas nuevas investigaciones, al resituar el contexto en el que se desarrollan las reformas institucionales, reconstruyen las arenas partidarias y parlamentarias de discusión, y escapan así de la trampa de considerar al sistema electoral como variable independiente. En lo que respecta a la derogación, se destaca no solo la importancia concedida al rol de los actores no partidarios (Organizaciones No Gubernamentales, Universidades, medios de comunicación), sino también al estudio de las divisiones al interior del justicialismo provincial. Cabe resaltar que logran despegarse de las limitaciones derivadas del rational-choice que, aplicado al análisis político, presupone que los actores siempre actúan maximizando su propio interés, entendido como incremento de su cuota de poder. Finalmente, incorporan la

\footnotetext{
${ }^{9}$ La Ley 10.802 incluye disposiciones referidas a las listas partidarias presentadas ante el Tribunal Electoral Provincial sin distinguir entre primarias y generales. Esto tuvo como resultado que la distribución final de escaños perjudicara a las candidatas, generalmente ubicadas en los terceros lugares.
} 
dimensión simbólica de la política, ello permite complejizar nuestra comprensión de los procesos de reforma institucional.

Por último, el tercer grupo de contribuciones se vincula con distintas concepciones acerca de lo regional y la regionalización. Así, encontramos algunas que abordan la regionalización emprendida por el Estado santafesino luego de la asunción a la gobernación del Frente Progresista Cívico y Social (FPCyS) en el 2007 y que destacan los conflictos políticos que esa iniciativa genera, en especial por parte de los senadores justicialistas (Berdondini, Pinillos y Signorelli, 2015). También hay otros estudios que explican cómo las fracturas socioeconómicas de base regional se traducen a nivel del sistema político (Viana, 2014). ${ }^{10}$ Finalmente, en los últimos años se efectuaron investigaciones que dan cuenta de las bases regionales del voto políticopartidario. Contrariamente a lo esperado, dejan en claro que ninguna de las fuerzas partidarias santafesinas posee actualmente bases regionales sólidas que se mantengan a lo largo del tiempo (Scaramella y Contursi Reynoso, 2019). ${ }^{11}$

Para cerrar este apartado destacamos que, en los últimos años, la expresión "política provincial" ha dejado lugar a la noción de "política subnacional", lo cual da cuenta de una tendencia estructural que cruza a las distintas disciplinas, vinculada con la creciente importancia de lo local. En segundo lugar, que la competencia interpartidaria en distintas arenas electorales ha empezado a definirse en términos de "coordinación estratégica multinivel"; lejos de ser una mera transformación en el nombre, señala la incorporación de nuevas perspectivas teóricas asociadas al avance de metodologías cuantitativas en nuestra región, en especial en el campo de la ciencia política (Rocha Carpiuc, 2013).

\section{Partidos, liderazgos y élites políticas en Santa Fe}

La producción sobre actores partidarios de la provincia se ha multiplicado en los últimos años. Desde una diversidad de disciplinas y enfoques teórico-metodológicos, numerosos análisis han abordado el devenir del sistema partidario y sus protagonistas desde la recuperación democrática. Podemos identificar un primer conjunto de trabajos que se ocuparon de estudiar alguna de las distintas fuerzas que integran el sistema político santafesino (PJ, UCR, PS, PDP y PRO, entre las de mayor relevancia). En este grupo predomina el abordaje de tipo historiográfico, aunque también se recuperan la mirada sociohistórica y el análisis político. Así, estos estudios han indagado en la dinámica organizacional de los partidos (Guberman Marcipar, 2004; Maina, 2008; Gómez y Recio, 2013; Lacher, 2015; Rinaldi, 2019), en la conformación de distintas

\footnotetext{
${ }^{10}$ En esta incipiente línea se hace explícito un presupuesto compartido por la amplia mayoría de los trabajos; esto es: las diferencias entre los departamentos del norte, centro y sur santafesinos y su relevancia a nivel de la competencia político-partidaria.

${ }^{11}$ Esta es una conclusión muy significativa ya que era un supuesto aceptado hasta el momento que el centro-norte provincial tendía a inclinarse a favor del justicialismo, mientras que el sur, luego de 1983, favorecía a las fuerzas de la oposición.
} 
fracciones y liderazgos internos ${ }^{12}$ y en el modo en el que cada fuerza buscó construir un vínculo representativo frente a la ciudadanía (Ramos, 2011; Lascurain, 2014; Prado, 2019). La mayoría de ellos examinan los partidos en el nivel provincial, aunque son todavía escasas y recientes las indagaciones a nivel municipal/comunal. En esta línea cabe mencionar un estudio sobre la UCR en la ciudad de Santa Fe (Landau, 2020), otro referido al Movimiento de Afirmación Vecinalista de la ciudad de Rafaela (Tonon, 2019) y los análisis del PS como fenómeno inicialmente arraigado en la ciudad de Rosario (Guberman Marcipar, 2004; Suárez, 2018).

Otro grupo de pesquisas -que se ubica más claramente en una matriz de sociología política, y con un menor desarrollo que el anterior- se ha focalizado en el examen de las dirigencias o élites partidarias que ocuparon, a su vez, posiciones de gobierno. Aquí se inscriben los trabajos que indagan en la composición de elencos gubernamentales, sean del ámbito ejecutivo (Lascurain, 2018) o legislativo (Perri, 2019), como asimismo aquellos que analizan las características y el comportamiento de la dirigencia política en tanto que integrante de "grupos políticos" (Landau, 2020). Estas investigaciones utilizan como herramienta metodológica típica el análisis de trayectorias y el estudio prosopográfico de los grupos. Como insumo teórico, permiten auscultar los múltiples espacios por los que se mueven individuos que pertenecen a un colectivo determinado, prestando especial atención al tejido de vínculos interpersonales de confianza y lealtad. Además, descentran el foco usualmente situado en el "partido" o en el "gobierno" para explorar ámbitos atravesados por prácticas políticas -como las instituciones educativas, las organizaciones de la sociedad civil, entre otros- y advertir, así, los vínculos más o menos fuertes entre unos y otros.

Por último, encontramos investigaciones de corte politológico cuya unidad de análisis es el sistema político-partidario, y que fijan la mirada en coyunturas electorales específicas a nivel provincial (Delgado y Cherny, 2004; Delgado, 2006), o municipal (Cherny, 2003; Pinillos y Sassaroli, 2005; Bailone, 2013). De manera similar a los estudios sobre sistemas electorales, los trabajos recorren una preocupación teórica común atenta a las transformaciones en el vínculo de representación política, especialmente para el período posterior a la llamada "crisis de representación partidaria" (Torre, 2003) del 2001. Sin embargo, hacen especial hincapié en analizar los modos novedosos bajo los cuales las candidaturas se presentan frente a la sociedad, los procesos de personalización de la política y la centralidad que aún conservan las estructuras partidarias en los contextos electorales. Además, reparan en el examen de la expresión ciudadana, esto es: la debilidad de las identidades políticas tradicionales para estructurar el voto, la selectividad de la opción electoral y la volatilidad entre procesos electorales y entre categorías dentro de una misma elección. También identificamos estudios que abordan el comportamiento de los partidos y los liderazgos provinciales abarcando procesos históricos de mediano plazo, como el período de la "transición democrática" (Maina, 2014) o las transformaciones en las "lógicas de acumulación

\footnotetext{
12 En particular la Renovación Peronista (Baeza Belda, 2010; Maina, 2016), la línea reutemannista (Lascurain, 2019) y el kirchnerismo en su versión local (Ramos y Vaschetto, 2019).
} 
política" de las principales fuerzas a lo largo de la década del noventa (Geary y Robin, 2005).

En este conjunto podemos identificar algunos aspectos comunes y ciertas limitaciones y áreas de vacancia que podrían recuperarse para el desarrollo de futuras pesquisas. En cuanto a lo primero, advertimos que la totalidad de la producción relevada se sitúa en el análisis de la política santafesina, pero dialoga de manera más o menos explícita y reflexiva con preguntas, procesos e indagaciones empíricas surgidas de investigaciones situadas en la escala nacional o en otras jurisdicciones provinciales. Podemos mencionar como ejemplo el debate acerca de si hubo o no una "Renovación Peronista" en la provincia; la pregunta por la capacidad de las estructuras partidarias provinciales para moldear o limitar la acción de nuevos "liderazgos de audiencia"; o el interrogante sobre la manera en que el estudio del espacio local puede aportar a una mejor comprensión del modo en que se tejen los lazos hacia el interior de los grupos políticos. ${ }^{13}$ Esta preocupación se comprende al tomar en cuenta el contexto de producción de gran parte de las obras citadas. En efecto, el interés por reflexionar en torno al problema de las "escalas" en el análisis de los fenómenos políticos recientes data de comienzos del nuevo siglo -al menos en Argentina (Águila, 2015)-, momento a partir del cual se registra la mayor parte de las investigaciones relevadas en este segundo apartado. Esto es consistente con el hecho de que -como mostramos en el primer eje- la producción académica desarrollada durante los años noventa se concentró, principalmente, en el estudio del régimen electoral.

Debemos reconocer, sin embargo, como una primera limitación el hecho de que en este concierto de trabajos priman aquellos abordajes que contemplan la influencia o la vinculación de lo "nacional" con lo "provincial" y, en menor medida, los que parten de escalas más pequeñas, para articularlas luego con fenómenos de más amplio alcance. Peor aún, sigue presente cierta pretensión epistemológica por indagar en qué medida y sobre qué elementos los fenómenos estudiados a escala provincial "se acercan" o "se alejan" de aquellos enfocados en la escala nacional, como así también la tendencia a aplicar determinados conceptos o teorías más generales al estudio empírico del caso provincial o municipal. Precisamente, la consideración de la "escala" como una variable a examinar hace posible la pregunta por aquello que el análisis del caso en profundidad y a menor escala aporta, discute o incluso refuta de las conceptualizaciones más generales y enfocadas -usualmente- en lo "nacional".

Una segunda cuestión a señalar es la acotada producción existente referida a liderazgos y élites partidarias de la provincia de Santa $\mathrm{Fe}$ en la historia reciente. Un

\footnotetext{
13 Un relevamiento más ajustado a los interrogantes que posibilita una perspectiva de historia regional debería incluir las producciones que recuperan problemas y cuestiones que involucran a Santa Fe y a otras jurisdicciones provinciales, locales o transnacionales. Con esto nos referimos no solamente a los trabajos de índole comparativa entre distintos distritos (abundantes en ciencia política), sino a aquellos cuyas preguntas de origen involucran espacios regionales como la llamada "Región Centro". Por cuestiones de espacio y por tratarse de un relevamiento que, con estas características, no cuenta con antecedentes similares publicados, decidimos no ampliar la indagación hacia este tipo de estudios, aunque bien puede formar parte de esfuerzos posteriores que reflexionen sobre la escala regional y el modo en el que se produce conocimiento desde este enfoque.
} 
nombre insoslayable en este sentido es el de Carlos Reutemann, gobernador en dos oportunidades (1991-1995 y 1999-2003), senador nacional desde 2003 hasta 2021 cuando falleció- y figura controversial en la política provincial, cuya acción política y modos de gobernar han sido objeto de la atención de una escasa cantidad de investigaciones. ${ }^{14}$ En la misma línea, sería auspicioso que futuros abordajes abran el camino para identificar las características, los modos de reclutamiento y las formas de reproducción de las dirigencias o elites políticas provinciales y municipales. El hecho de que, aún contra el impedimento constitucional de la reelección inmediata del gobernador, se constituyan y gocen de gran influencia importantes liderazgos a lo largo de muchos años habilita a debatir y reflexionar más profundamente sobre las específicas modalidades de acción política desplegadas en este espacio regional.

Por último, un tercer ámbito de relativa vacancia refiere a las líneas o corrientes internas de partidos no peronistas. Esto es notorio en relación con la UCR santafesina dada la relevancia histórica que ha tenido, por ejemplo, la corriente de la Franja Morada hacia adentro y hacia afuera de la estructura partidaria, y la potencia de sus liderazgos surgidos del núcleo de militantes y dirigentes, no solo en el espacio provincial sino también en el nacional.

\section{Gobiernos, partidos y actores sociales}

El último grupo relevado está constituido por un heterogéneo conjunto de producciones, sin un claro predominio de una disciplina o de un eje teórico o metodológico articulador. Lo componen trabajos que indagan en los vínculos existentes entre, por un lado, el polo más "tradicional" de actores objeto de estudio de la historia política y la politología (partidos, gobiernos y poderes del Estado) y, por el otro, grupos y organizaciones de la sociedad civil que participaron y participan de la política santafesina: sindicatos y movimientos sociales, ${ }^{15}$ entre otros. ${ }^{16}$

El campo temático con mayor continuidad dentro de este recorte ha sido aquel preocupado por el estudio del sindicalismo santafesino (particularmente de la región

\footnotetext{
${ }^{14}$ Recién en 2003 aparecen las primeras producciones que analizan el surgimiento de la figura de Reutemann y su indiscutible influencia política en la provincia. Al respecto, se pueden consultar Nicolás Cherny (2003), Hugo Ramos (2011) y Cecilia Lascurain (2019).

${ }^{15}$ En este punto, cabe señalar la existencia de importantes trabajos historiográficos referidos a la formación y trayectoria del movimiento de Derechos Humanos en la provincia. Decidimos no incluir una revisión de esta extensa literatura por cuestiones de recorte temático, ya que adoptan una perspectiva anclada en el estudio de los movimientos sociales, sin ahondar en profundidad en su participación en la competencia político-partidaria.

${ }^{16}$ Antes de avanzar sobre las líneas de análisis que han tenido una mayor continuidad en el tiempo, queremos destacar un trabajo colectivo relativamente temprano que abordó desde una perspectiva local la crisis y los saqueos producidos a finales del mandato de Alfonsín en el año 1989. Nos referimos al volumen A 10 años de los saqueos en Rosario. Crisis social, medios y violencia (AAVV, 1999). Esta obra reúne aportes de diversas disciplinas con el propósito de reconstruir y analizar exhaustivamente la coyuntura social, política y económica en la que tuvo lugar uno de los más impactantes estallidos sociales de la historia local y provincial.
} 
sur) y sus vínculos con el mundo partidario de la provincia. Dentro de este marco amplio, es posible distinguir dos líneas de análisis. ${ }^{17}$

En primer lugar, podemos identificar un conjunto de trabajos que indagan la relación sindicatos-PJ durante los años ochenta (Maina, 2005, 2008, 2016; Baeza Belda, 2010; Lacher, 2015). Estas producciones de disciplinas diversas dan cuenta, en distinto grado, del rol central ocupado por el sindicalismo peronista al interior del PJ durante los primeros gobiernos provinciales posdictadura. Todos constatan el progresivo debilitamiento del poder político sindical, expresado en la pérdida de posiciones al interior del partido y en ámbitos legislativos provinciales y nacionales. Sin embargo, el foco no está puesto en los sindicatos; no se reconstruye el proceso desde la perspectiva de las organizaciones gremiales, sino desde el partido. Asimismo, se dice poco de los vínculos sindicales con otras fuerzas políticas, o su accionar en otras escalas.

Por otro lado, es posible rastrear otra línea de investigación que ha contado con una relativa permanencia. El primer exponente lo constituye el temprano aporte de Arturo Fernández (2016, publicado originalmente en 1993), elaborado desde una perspectiva de análisis politológica y al calor de las rápidas transformaciones económicas y sociales experimentadas en Argentina a principios de los años noventa. Aquí el autor traza un "mapa" del mundo sindical rosarino, e identifica sus principales corrientes internas y las diferentes estrategias de vinculación con lo político-partidario que estas esgrimen. Así, procura reconstruir la lógica política propia del sindicalismo rosarino "desde abajo hacia arriba", resaltando las particularidades locales y su lógica interna, aunque sin desconocer el contexto general más amplio que le da sentido. Fernández propone luego una tipología para pensar los vínculos sindicatos-partidos, construida fundamentalmente desde los intereses y estrategias desplegados por el polo gremial.

Esta línea fue recuperada posteriormente por Gloria Rodríguez (2007), quien desde la historiografía da cuenta de los principales ciclos de protesta sindical de la provincia desde los noventa hasta comienzos del siglo XXI, deteniéndose en el desarrollo de los nuevos repertorios de acción. Se destacan asimismo las particularidades señaladas anteriormente por Fernández (2016), que remiten a la existencia, en la región sur de la provincia, de prácticas y conducciones sindicales más combativas que aquellas visibles a nivel nacional. En la misma línea, un conjunto de cientistas sociales ha publicado un tomo colectivo que continúa estas indagaciones (Iglesias, Lucca, Ferri y Venticinque, 2016). Con algunas excepciones, todos los trabajos focalizan en períodos recientes (2003-2007), y reconstruyen los ciclos de conflictividad y movilización sindical, sus estrategias de coordinación y negociación, y las formas en que participaron en el ámbito político electoral, tanto a escala local (Rosario) como

\footnotetext{
${ }^{17}$ Cabe mencionar otros trabajos sobre el mundo sindical santafesino pero que no forman parte de estas líneas de abordaje. Nos referimos a los aportes de Cecilia Tonon (2012) y Marcelo Andelique y Cecilia Tonon (2016). El primero da cuenta de la experiencia del sindicato de empleados municipales de la ciudad de Rafaela entre 2005 y 2008; el segundo aborda a los sindicatos docentes santafesinos durante los años ochenta.
} 
provincial. Es de destacar que los sindicatos ocupan el centro del análisis, y que este no se limita a aquellas organizaciones identificadas con el peronismo.

Un segundo tema de preocupación que ha dado pie a una producción importante, aunque fragmentaria y discontinua, refiere a la cuestión de las reformas policiales en la provincia. ${ }^{18}$ Comenzamos con el temprano aporte de Fernando Rosúa (1998), quien fuera parte del Gobierno de Jorge Obeid. Su escrito, de carácter descriptivo, da cuenta del diagnóstico compartido por la dirigencia política provincial en ese momento: la autonomía de la Policía provincial respecto de la conducción política, y la consecuente ausencia de una verdadera política de seguridad. Unos años después, en un trabajo de corte jurídico, Gustavo González (2007) identifica dos grandes "polos" en los proyectos de reforma policial presentados hasta entonces: "garantismo" vs "punitivismo". El autor avanzó en una periodización de la problemática, y ubica a mediados de los noventa el ingreso definitivo de la seguridad pública como tema central en la agenda política provincial. González detecta la ausencia de proyectos de reforma globales, en favor de una sucesión de transformaciones puntuales arduamente negociadas entre el gobierno y las fuerzas policiales provinciales, que una vez más se presentan como un actor con un elevado grado de autonomía. En otro aporte, Gustavo González, Augusto Montero y Máximo Sozzo (2008) ${ }^{19}$ exploran la misma cuestión desde una perspectiva sociológica, a partir de la cual indagan en las opiniones del personal policial y su posicionamiento frente a los intentos reformistas desplegados por los gobiernos provinciales, y detectan, en líneas generales, un marcado rechazo a ellas. En conjunto, estos trabajos denotan las dificultades experimentadas por el sistema político provincial para abordar la problemática de la seguridad y ejercer un control genuino sobre la estructura policial, problema que resurgirá de forma recurrente hasta la actualidad.

Otra línea reciente de investigación a destacar refiere a la Legislatura provincial, su funcionamiento interno y su posición en el marco más amplio de la disputa política provincial. Aunque los relevamientos sobre conformación y comportamiento legislativo aparecen ocasionalmente en contribuciones relativas a otros temas (sistema político y partidos, por ejemplo), en general se limitan a dar cuenta de la variación en la composición partidaria de las Cámaras y del papel jugado por ellas en relación con el gobierno provincial de turno. Solo a partir de 2010 encontramos otros estudios más sistemáticos, de corte politológico, que ubican al Poder Legislativo en el centro de la escena. Un aporte temprano lo constituye el de Lourdes Lodi (2010); preocupada por explicar el proceso de derogación de la ley de lemas, la autora identifica a la Legislatura como un espacio de disputa fundamental para comprender el devenir del proyecto de reforma, haciendo hincapié en la necesidad de dar cuenta de la dinámica interna del

\footnotetext{
${ }^{18}$ No nos ocupamos de analizar las especificidades de las reformas policiales, sino de recuperar los trabajos que reconstruyen el proceso político que da paso a la problematización de la seguridad como tema de agenda política, dando cuenta del papel jugado por diversos actores del sistema político provincial.

${ }^{19}$ Estos autores han desarrollado una extensa producción académica en temas vinculados con la seguridad pública. Aquí solo recuperamos los trabajos relativos a nuestra área de interés.
} 
bloque mayoritario y del rol jugado por las comisiones en el avance o fracaso de este tipo de iniciativas.

Una investigadora que avanza en el análisis del funcionamiento legislativo provincial es Cecilia Rodríguez (2016), quien reconstruye las redes de cooperación legislativa existentes en la Legislatura entre 1995 y 2007. La politóloga señala el peso predominante de la legislación propuesta por el oficialismo provincial entre aquella que es efectivamente aprobada y el nivel bajo de cooperación legislativa entre partidos, aunque detecta la existencia de redes de cooperación. Estas se ponen en juego en coyunturas específicas para facilitar la sanción de leyes patrocinadas por dos o más fuerzas, y es fundamental la participación de alguna fracción del oficialismo provincial para que la iniciativa tenga éxito.

Este último punto resulta de particular interés en tanto devela una de las características fundamentales del juego legislativo provincial, detectada por estos trabajos: a contramano de lo que el sesgo mayoritario de su composición podría sugerir, la Legislatura provincial ha ejercido en múltiples ocasiones el papel de poder de veto de las principales iniciativas gubernamentales. Esto se explica por la fragmentación interna de los partidos provinciales, que torna decisiva la capacidad de disciplinamiento de la bancada propia ejercida por el gobernador de turno. Asimismo, esta división interna posibilita el juego "cruzado" entre fracciones de distintos partidos. Estas características de la dinámica legislativa santafesina son expuestas con toda claridad por Augusto Abdulhadi (2018). El autor estudia los mecanismos a partir de los cuales se produce el cambio en la composición de la Corte Suprema provincial entre 1983 y 2011. Dada la fragmentación de los partidos en la legislatura y las exigencias constitucionales para nombrar o remover jueces, Abdulhadi demuestra que la negociación entre fracciones (en este caso, fundamentalmente del PJ y la UCR) resulta crucial para el éxito o fracaso de las iniciativas gubernamentales.

Lo que este pequeño grupo de investigaciones repone, entonces, es la centralidad de la negociación a nivel legislativo en la política provincial. Lejos de lo que el sesgo mayoritario establecido constitucionalmente podría sugerir, la Legislatura santafesina no ha sido una mera "escribanía" oficialista, sino un espacio de negociación fundamental en el que han pesado los liderazgos fraccionales. Queda pendiente, en este mismo sentido, avanzar en trabajos que examinen las trayectorias políticas de los legisladores provinciales, especialmente senadores, quienes se presentan en muchos de ellos como actores fundamentales del escenario provincial.

\section{Reflexiones finales}

En este artículo presentamos una apretada síntesis de los aportes que aborda la historia política reciente en la provincia de Santa Fe, desde 1983 hasta la actualidad, provenientes de diversas disciplinas. De la exposición precedente sistematizamos una 
serie de conclusiones que esperamos sean de utilidad para investigadores del campo. ${ }^{20}$ A diferencia de la división en áreas temáticas, utilizamos aquí un abordaje cronológico, por décadas, porque entendemos que permite una mejor comprensión de lo fragmentario y disperso que es nuestro conocimiento de la historia política santafesina. En este sentido, los años ochenta han sido escasamente estudiados. En la línea asociada a los sistemas electorales prácticamente no se registran investigaciones. Si atendemos al análisis de los actores del sistema político partidario, el PJ ha sido el que ha despertado mayor interés, probablemente por ser el partido victorioso en las elecciones fundacionales de 1983 y por ciertas particularidades vinculadas al proceso renovador, a mitad de los años ochenta. Por el contrario, el estudio de otras organizaciones partidarias se encuentra en sus inicios. Idénticas conclusiones podemos extraer en lo referente a otros actores sociopolíticos, como universidades, sindicatos y organizaciones empresariales. Aún la observación de la Legislatura provincial y de la conformación del Poder Judicial en estos años clave ha despertado hasta el momento escaso interés. Los principales aportes sobre la década de la transición provienen del enfoque de los movimientos de defensa de los derechos humanos -que no abordamos aquí- y del esfuerzo desplegado por investigaciones puntuales. ${ }^{21}$

Los años noventa, en cambio, presentan un mayor número de trabajos, a la vez que se observa una amplia diversificación temática. Aquí, lo escrito acerca del sistema electoral ocupa un lugar central, con las limitaciones y problemas ya señalados. Fuera de esta línea, se destacan las investigaciones centradas en los partidos políticos en coyunturas eleccionarias, que han aportado un importante acervo de conocimientos sobre la evolución de aquellos a lo largo de la década. Aquí cobran relevancia los insumos de la ciencia política, que han permitido trabajar desde una escala provincial las problemáticas ligadas a las transformaciones en las relaciones de representación, la construcción de liderazgos y la conformación de "democracias de audiencia". Asimismo, gracias a estos escritos podemos identificar las estrategias adaptativas implementadas en las organizaciones partidarias, así como avanzar en la comprensión de su vida interna. Sin embargo, los aportes tienen un claro sesgo a favor del PJ y son escasas las investigaciones en torno a la UCR, el PS o el PDP. También se observa una notoria vacancia en el estudio de los actores sociopolíticos, con el agregado de que, en una década en que los mass media empiezan a ocupar un rol central en la esfera política, no se identificó ninguna producción que atendiera a cuáles son esos medios en Santa Fe y a cómo operan en la arena política provincial. En este sentido: ¿se registran los mismos fenómenos que a nivel nacional? Si la respuesta es afirmativa, ¿por qué todos los trabajos, a la vez que destacan el fenómeno de "mediatización de la política" señalan el enraizamiento partidario de los nuevos liderazgos y la todavía importante presencia de las identificaciones partidarias? Se observa aquí cierta tendencia a asumir de forma acrítica fenómenos que sucedieron en la escala nacional

\footnotetext{
20 La utilización de la noción de "campo" es solo un recurso narrativo. Las características de la producción sobre la historia política de Santa Fe que detallamos en el artículo dan cuenta de que, si es que existe, este campo se encuentra aún en formación.

${ }^{21}$ Con matices, y como desarrollamos a lo largo del artículo, esta afirmación se extiende a las décadas más recientes.
} 
que son "traducidos" a la escala provincial sin investigaciones empíricas que las respalden. Similar apreciación cabe realizar respecto de la recuperación de la categoría de clientelismo utilizada por varios de estos aportes: hasta el momento no existe ninguna investigación que dé cuenta de su relevancia en el ámbito de la provincia; sin embargo, se asume que es una estrategia implementada de forma reiterada por el justicialismo. Es necesario avanzar en exploraciones empíricas que estudien las relaciones entre partidos y ciudadanos, en especial los vínculos personalizados que no necesariamente se circunscriben a la provisión material, sino que movilizan también valores morales y simbólicos (en este último sentido, es sintomática la ausencia de indagaciones de corte antropológico). Asimismo, es prioritario avanzar en el análisis de las élites políticas santafesinas, en especial en términos de su pertenencia a "grupos políticos" con características particulares, lo que nos permitiría tener una noción más clara acerca de su composición.

Finalmente, el período que se abre con el nuevo siglo replica en general los problemas señalados, pero con algunas salvedades. En primer lugar, los enfoques que incorporan las teorías de género, si bien minoritarios, abren un campo novedoso para la exploración empírica. En segundo término, la derrota del justicialismo en el 2007 habilitó la ampliación de la agenda de investigación y son más numerosos los trabajos que analizan en profundidad a otras organizaciones políticas o coaliciones. Sería deseable sumar nuevas pesquisas, en particular en torno al PRO. También se observa una mayor atención al Poder Legislativo y a diversas problemáticas, en general ligadas a los frustrados intentos de reforma constitucional, que han permitido sumar al Poder Judicial dentro del área de investigaciones. En la misma línea, los estudios sobre "lo regional" y la regionalización seguramente permitirán poner en discusión algunos supuestos, como la base territorial del voto de los partidos provinciales. Sin embargo, los aportes centrados en los actores sociopolíticos siguen siendo, en general, los grandes ausentes.

Antes de concluir, es necesario realizar un comentario adicional. Los estudios sobre la estructura de la competencia política identifican claramente un esquema bipolar que se extiende desde 1995 hasta 2007. Asimismo, los escasos trabajos que abordan la década de los ochenta señalan al bipartidismo -con un tercer partido con capacidad de obtener escaños- como rasgo central. Para el período posterior al 2007, todas las contribuciones asumen que desde el 2011 la provincia presenta tres grandes fuerzas políticas con capacidad de acceder al poder (PJ, FPCyS y PRO-Cambiemos). Estos cambios en la estructura de la competencia, sin embargo, no han operado como insumos para repensar la periodización de la historia reciente santafesina, que se sigue articulando en términos de la historia política nacional o de acuerdo con el mandato de los gobernadores.

El estado del arte realizado sugiere que el campo de la historiografía recién empezó a indagar en la etapa pos 1983 bien avanzado el siglo XXI. Es claro que la agenda investigativa no incluía, hasta hace muy poco tiempo, las problemáticas recientes. Saludamos, en este sentido, el cambio que se observa en los últimos años. 
Lascurain, M.C.; Ramos, H. D. y Vaschetto, M. La historia política de Santa Fe desde la transición democrática (19832020): aportes, líneas de investigación y vacancias

\section{Referencias bibliográficas}

1. AA.VV. (1999). A 10 años de los saqueos en Rosario. Crisis social, medios y violencia. Universidad Nacional de Rosario.

2. Abdulhadi, A. (2018). Cortes judiciales y democracia. Estrategias y mecanismos para los cambios políticos de jueces en las provincias argentinas (1983-2011) [tesis de doctorado, Universidad Nacional de San Martín, tesis no publicada].

3. Águila, G. (2015). Las escalas de análisis en los estudios sobre el pasado reciente: a modo de introducción. Avances del Cesor, XII (12), 91-96. https://doi.org/10.35305/ac.v12i12.430

4. Andelique, M. y Tonon, M. C. (2016). Una aproximación a las acciones colectivas de los docentes santafesinos frente a las políticas educativas (1983-1989). De Prácticas y discursos, 5 (6), 1-19. https://doi.org/10.30972/dpd.56701

5. Baeza Belda, J. (2010, 15 de septiembre). La Renovación Peronista: el caso de la provincia de Santa Fe (1983-1988) [ponencia]. XIV Encuentro de Latinoamericanistas Españoles. Santiago de Compostela, España.

6. Bailone, J. (2013). Las características de la representación política en la ciudad de Santa Fe. Un análisis del período 2009-2011 [trabajo final de carrera, Universidad Nacional del Litoral, trabajo no publicado].

7. Berdondini, M., Pinillos, C. y Signorelli, G. (2015). Territorio y actores políticos en el proceso de regionalización de la Provincia de Santa Fe. Una disputa por "los caminos de la gente". Cartografías, 0 (1), enero, 29-46. https://doi.org/10.35428/cds.v0i1.2

8. Calvo, E. y Escolar, M. (2005). La nueva política de partidos en la Argentina. Prometeo.

9. Catalin, C. (2018). Boleta única por categoría o Boleta única electrónica: una mirada desde la experiencia santafesina. En G. Mutti y A. Torres (Comps.) Los procesos electorales en perspectiva comparada (pp. 262-290). UNR Editora.

10. Cherny, N. (2003). La representación frágil: de la polarización al desapego. Elecciones y política en la ciudad de Rosario. En I. Cheresky y J-M. Blanquer (Comps.) De la ilusión reformista al desencanto ciudadano. Las elecciones en Argentina 1999-2001 (pp. 159-186). Homo Sapiens. 
11. Delgado, M. S. (2006). Frente a Frente. Alianzas electorales, reforma institucional y recomposición política en Rosario y Santa Fe (2004-2006). En I. Cheresky (Comp.) La política después de los partidos (pp. 241-275). Prometeo.

12. Delgado, M. S. y Cherny, N. (2004). Coaliciones transversales y ciudadanía selectiva. Las elecciones de 2003 en Rosario y Santa Fe. En I. Cheresky y I. Pousadela (Eds.) El voto liberado. Elecciones 2003: perspectiva histórica y estudio de casos (pp. 167-191). Homo Sapiens.

13. Díaz, C. (1996). "De eso no se habla". Representación y ley de lemas: un análisis político. En J. C. Venecia (Comp.) Ley de lemas: alternativas de reforma políticoelectoral en Santa Fe (pp. 63-76). Homo Sapiens.

14. Fernández, A. (2016). Tipos de vinculación entre sindicatos y partidos en Rosario. En E. Iglesias, V. Venticinque, J. B. Lucca y M. N. Ferri (Comps.) Partidos políticos y sindicatos en Rosario (pp. 43-61). UNR Editora. (Edición original en 1993).

15. Fernández, E. (2017). Competencia electoral en los municipios de la Provincia de Santa Fe: un análisis sobre los efectos del Doble Voto Simultáneo y las Primarias Abiertas Simultáneas y Obligatorias (1991-2015). Studia Politicae, 42, 107-136. https://doi.org/10.22529/sp.2017.42.04

16. Ferro, L. (2003, 10 de diciembre). Género y participación política en Santa Fe. Ser, estar y actuar [ponencia]. III Jornadas de Sociología de la Universidad Nacional de la Plata. La Plata, Argentina.

17. Franco, M. y Levín, F. (Comps.) (2007). Historia reciente. Perspectivas y desafíos para un campo en construcción. Paidós.

18. Geary, M. y Robin, S. (2005, 15 de noviembre). Los partidos en el interior y el interior de los partidos. Peronismo y radicalismo en Santa Fe durante los '90 [ponencia]. VII Congreso Nacional de Ciencia Política. Córdoba, Argentina.

19. Gómez, S. y Recio, M. (2013). La llegada al poder del Frente Progresista Cívico y Social en la Provincia de Santa Fe. Viejas disputas, nuevos desafíos (2007-2011). En S. Robin y A. Ford (Comps.) Representación y participación democrática en los espacios locales (pp. 313-330). Ediciones Del Revés.

20. González, G. (2007). Reforma policial y política: Un complejo entramado de compromisos, resistencias y condiciones de posibilidad. Urvio, Revista Latinoamericana de Seguridad Ciudadana, 2, 154-163. https://doi.org/10.17141/urvio.2.2007.1067 
Lascurain, M.C.; Ramos, H. D. y Vaschetto, M. La historia política de Santa Fe desde la transición democrática (19832020): aportes, líneas de investigación y vacancias

21. González, G., Montero, A. y Sozzo, M. (2008). ¿Reformar la policía? Representaciones y opiniones de los policías en la provincia de Santa Fe. En E. Bohoslavsky, L. Caimari y C. Schettini (Eds.) La policía en perspectiva histórica. Argentina y Brasil (del siglo XIX a la actualidad). CDRom.

22. Guberman Marcipar, L. C. (2004). El Partido Socialista Popular en Rosario 19891995 [tesis de maestría, Universidad de Buenos Aires, tesis no publicada].

23. Iglesias, E., Venticinque, V., Lucca, J. B. y Ferri, M. N. (Comp.) (2016). Partidos políticos y sindicatos en Rosario. UNR Editora.

24. Lacher, A. (2015). Desindicalización del Partido Justicialista de la Provincia de Santa Fe (1983-1989) [trabajo final de carrera, Universidad Nacional del Litoral, trabajo no publicado].

25. Landau, M. (2020). Sociabilidad y grupos políticos. El radicalismo universitario en la ciudad de Santa Fe, 2007-2015. Estudios Políticos, 58, 263-284. https://doi.org/10.17533/udea.espo.n58a12

26. Lascurain, M. C. (2014). De políticos honestos y corruptos: el affaire Vanrell y la mutación de la representación en el justicialismo santafesino (1990-1991). Revista PolHis, 7(14), 375-417.

27. Lascurain, M. C. (2018). Acerca de las elites gubernamentales subnacionales. Los gobernadores y vicegobernadores peronistas de Santa Fe, Argentina (19832007). Revista Mexicana de Ciencias Políticas y Sociales, 63 (233), 307-332. https://doi.org/10.22201/fcpys.2448492xe.2018.233.57827

28. Lascurain, M. C. (2019). De outsider a conductor. Emergencia y consolidación del liderazgo de Carlos Reutemann en el peronismo santafesino (1989-1993). En A. Farji Neer, A. González, M. Greco y V. Le Borgne (Comps.) Las ciencias sociales en tiempos de ajuste (pp. 125-160). Universidad de Buenos Aires/Instituto de Investigaciones Gino Germani/Consejo Latinoamericano de Ciencias Sociales. https://doi.org/10.2307/j.ctvt6rm8t.10

29. Lodi, M. de L. (2010, 28 de julio). La Política de la Reforma Electoral en Santa Fe [ponencia]. V Congreso Latinoamericano de Ciencia Política. Buenos Aires, Argentina.

30. Maina, M. (2005, 20 de septiembre). Partidos, Sindicatos y Asociaciones. Santa $\mathrm{Fe}$, en el ocaso de la última dictadura militar [ponencia]. X Jornadas Interescuelas/Departamentos de Historia. Rosario, Argentina. 
31. Maina, M. (2008, 10 de diciembre). Una desorganización organizada: el justicialismo santafesino 1982-1987 [ponencia]. V Jornadas de Sociología. La Plata, Argentina.

32. Maina, M. (2014). Santa Fe hacia las elecciones de 1983. Partidos, identidades y elecciones en la construcción de la democracia. Papeles del Centro de Investigaciones, 4 (15), 119-141. https://doi.org/10.14409/p.v1i15.4398

33. Maina, M. (2016). Santa Fe. En M. Ferrari y V. Mellado (Comps.) La Renovación Peronista. Organización partidaria, liderazgos y dirigentes 1983-1991 (pp. 153176). Editorial Universitaria de Tres de Febrero.

34. Manin, B. (1996). Metamorfosis de la representación. En M. Dos Santos (Coord.) ¿Qué queda de la representación política? (pp. 9-40). Nueva Sociedad.

35. Mutti, G., Borello, R., Lodi, M. L. (2008, septiembre). Las formas de la política: régimen político y cambios institucionales en la Provincia de Santa Fe a partir de 2003 [ponencia]. I Jornadas de Ciencia Política de la Universidad Nacional de Entre Ríos. Paraná, Argentina.

36. O' Donnell, G. y Schmitter, P. (2010). Transiciones desde un gobierno autoritario. Prometeo.

37. Perri, M. E. (2012). La influencia de la ley de cupo en la representación descriptiva y sustantiva de las mujeres en el poder legislativo provincial. El caso de la Provincia de Santa Fe (1983-2011) [trabajo final de carrera, Universidad Nacional del Litoral, trabajo no publicado].

38. Perri, M. E. (2017). El proceso paritario en Santa Fe. En XIII Congreso Nacional de Ciencia Política, pp. 4837-4853.

39. Perri, M. E. (2019). Las trayectorias políticas en Santa Fe. Análisis de género de los legisladores provinciales (1983-2015). En XIV Congreso Nacional de Ciencia Política. Buenos Aires, 17-20 de julio, pp. 4474-4492.

40. Petracca, A. (1991). Ley de lemas y democracia representativa. UNR Editora.

41. Petracca, A., Mutti, G., Molteni, R., Ford, A. y Borello, R. (2003). Cambio Institucional y Agenda Pública. La provincia de Santa Fe en los años noventa. UNR Editora.

42. Pinillos, C. y Sassaroli, V. (2005, 15 de noviembre). Política subnacional y oposición en la Ciudad de Rosario [ponencia]. VII Congreso Nacional de Ciencia Política de la SAAP. Córdoba, Argentina. 
Lascurain, M.C.; Ramos, H. D. y Vaschetto, M. La historia política de Santa Fe desde la transición democrática (19832020): aportes, líneas de investigación y vacancias

43. Prado, A. (2019). El PRO en Rosario: original o copia [trabajo final integrador, Universidad Nacional de Rosario, trabajo no publicado].

44. Puig, L. (2000). Estudio y evaluación del sistema electoral de la Ley de Lemas en la Provincia de Santa Fe y propuesta de reforma electoral. Apoyo al Programa de Reforma Política, ARG/00/007. Programa de Naciones Unidas para el Desarrollo.

45. Ramos, H. (2011). Metamorfosis y crisis de representación. Las estrategias electorales del PJ santafesino en las elecciones provinciales de 1999 y 2003. Revista SAAP, 5(1), 157-187.

46. Ramos, H. y Vaschetto, M. (2019). La emergencia del kirchnerismo en Santa Fe (2003-2005). En G. Mutti y A. Torres (Comps.) Procesos electorales en perspectiva multinivel (pp. 279-300). UNR Editora.

47. Rinaldi, M. (2019). El origen del reutemannismo y la dinámica organizacional del PJ santafesino (1990-1991). Colección, 30 (2), 45-87.

48. Robin, S. (1994). Ley de lemas y dinámica del sistema de partidos en la provincia de Santa Fe. Estudios Sociales, 6 (1), 85-95. https://doi.org/10.14409/es.v6i1.2313

49. Robin, S. (2007). Régimen electoral y sistema de partidos: la influencia de la ley de lemas sobre el sistema de partidos en la provincia de Santa Fe [tesis de maestría, Universidad Nacional de Rosario, tesis no publicada].

50. Rocha Carpiuc, C. (2013). ¿Hacia una hegemonía del "modelo mainstream norteamericano"? Enfoques de la ciencia política en América Latina (2000-2012). Documento de Trabajo, CLACSO.

51. Rodríguez, G. (2007, 8 de agosto). Estudios sobre formas de resistencia en organizaciones gremiales de Rosario y su región. Del neoliberalismo a la salida de la convertibilidad [ponencia]. $8^{\circ}$ Congreso Nacional de Estudios del Trabajo. Buenos Aires, Argentina.

52. Rodríguez, C. (2016). Redes de cooperación legislativa a nivel subnacional: análisis de los casos de Río Negro y Santa Fe. América Latina Hoy, 73, 45-66. https://doi.org/10.14201/alh2016734566

53. Rosúa, F. (1998), La reforma policial en la provincia de Santa Fe. En CELS, LaS reformas policiales en Argentina (pp. 37-67). Centro de Estudios Legales y Sociales. 
Lascurain, M.C.; Ramos, H. D. y Vaschetto, M. La historia política de Santa Fe desde la transición democrática (19832020): aportes, líneas de investigación y vacancias

54. Rulli, M. y Centeno Lappas, F. (2006). Procesos de reforma política: la derogación de la Ley de Lemas en Santa Fe. Temas $y$ debates, 12. https://doi.org/10.35305/tyd.v0i12.121

55. Scaramella, C. y Contursi Reynoso, A. (2019). Segmentación estratégica multinivel: el caso de Santa Fe en las elecciones de 2017. En G. Mutti y A. Torres (Comps.) Procesos electorales en perspectiva multinivel (pp. 185-198). UNR Editora.

56. Suárez, F. (2018). El Partido Socialista Popular: orígenes, organización y tradiciones políticas (1972-1982) [tesis de maestría, Universidad Nacional de Mar del Plata, tesis no publicada].

57. Tibaldo, A. (2013, 17 de julio). La fragmentación interna de los partidos políticos en Santa Fe: ¿efecto (deseado) de los sistemas electorales adoptados? [ponencia]. XI Congreso Nacional de Ciencia Política. Paraná, Argentina.

58. Tonon, M. C. (2012, agosto). "Una carpa... una lucha", formas de acción colectiva de un sindicato municipal santafesino, 2005-2008 [ponencia]. VI Jornadas sobre Historia Reciente. Santa Fe, Argentina.

59. Tonon, M. C. (2019). Partidos Vecinales durante la transición democrática. El caso del Movimiento de Afirmación Vecinalista en la Provincia de Santa Fe, 1982-1983. Revista PolHis, 12 (24), 94-123.

60. Toppi, H. (2015). Poder de agenda, contexto y cambio institucional como proceso en Argentina: los casos de la Ley de Cuotas y la Ley de Lemas. Miríada, $7(11), 121-165$.

61. Torre, J. C. (2003). Los huérfanos de la política de partidos. Desarrollo Económico, 42 (168), 647-665. https://doi.org/10.2307/3455908

62. Viana, C. (2014). Relaciones entre el poder político de la Región Centro/Norte y el de la Región Sur en la Provincia de Santa Fe [tesina de licenciatura, Universidad Abierta Interamericana, tesina no publicada]. 